\title{
Treatment approaches towards physical activity - malnutrition - cognitive impairment
}

\author{
Marlene Troch
}

In this issue of the Magazine of European Medical Oncology (MEMO) we aim to draw your attention to some highly underestimated but common conditions in cancer patients and their influence on outcome and treatment strategies. These articles will probably change your daily practice.

Dr. Kiesl summarizes data on exercise and physical activity with regard to cancer prevention, management of (cancer) treatment-related side effects and the importance of rehabilitation therapy in oncology. He underlines the difficulties of interpreting current data but also the convincing impact of physical activity on side effects with a favorable side effect profile itself [1].

Mag. Beirer collected important information on definition, screening and treatment of malnutrition in cancer patients. She emphasizes the high prevalence and the need of using standardized screening tools in daily routine in order to identify all patients presenting with malnutrition. As malnutrition may also influence cancer outcome negatively, assessment of nutritional factors should be an obligatory part of managing cancer patients. This article also includes descriptive algorithms for diagnosis and treatment of malnutrition [2].

Finally, Drs. Hutterer and Oberndorfer provide a very detailed overview on cancer-related cognitive impairment including definitions, pathogenetic mechanisms and common risk factors. Furthermore, the authors again highlight the need of standardized screening tools such as validated neuropsychological tests to identify the individual patient's current condition and needs. Although there is still no defini-

PD Dr. M. Troch $(\bowtie)$

Outpatient oncologic Rehabilitation, Klinik Pirwarth in Wien, Brünner Straße 70, 1210 Vienna, Austria

marlene.troch@klinik-pirawarth.wien tive medical treatment for cognitive impairment, Drs. Hutterer and Oberndorfer inform about effective therapeutic options including cognitive behavioral interventions and specific cognitive rehabilitation [3].

In summary, these articles cover important conditions which are omnipresent in the management of cancer patients. Using standardized screening tools in daily routine appears to be crucial to identify patients' needs and will potentially allow us to offer individual treatment strategies and improve quality of life as well as outcome of cancer patients.

Conflict of interest M. Troch has received honoraria for lectures and articles from the following for-profit companies: Novartis, Astellas, Sandoz.

\section{References}

1. Kiesl D. Importance of performance status and physical activity in cancer patients. memo. 2021. https://doi.org/ 10.1007/s12254-021-00698-1.

2. Beirer A. Malnutrition and Cancer, Diagnosis and Treatment. memo. 2021. https://doi.org/10.1007/s12254-02100672-3.

3. Hutterer M, OberndorferS. Cognitiveimpairment in cancer patients and survivors-clinical presentation, pathophysiology, diagnosis and management. memo. 2021. https:// doi.org/10.1007/s12254-021-00690-9.

Publisher's Note Springer Nature remains neutral with regard to jurisdictional claims in published maps and institutional affiliations.

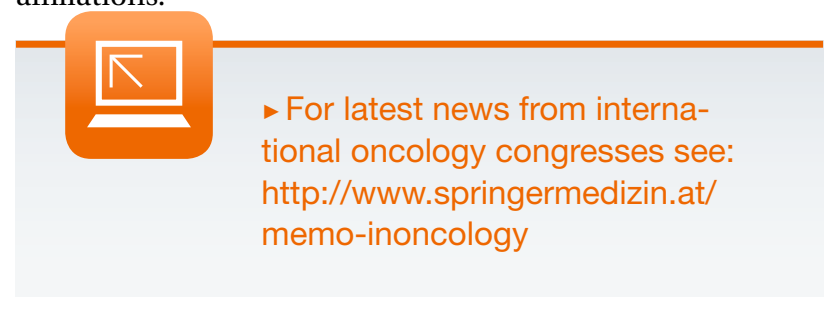

